\title{
Misleading Concepts about Mysterious Zero
}

\author{
Mohammad Azram* \\ Department of Science, Faculty of Engineering, IIUM, Kuala Lumpur 50728, Malaysia. \\ * Corresponding author. Tel.: +60166842042; email: azram50@hotmail.com \\ Manuscript submitted March 1, 2015; accepted June 8, 2015. \\ doi: 10.17706/ijapm.2015.5.3.185-191
}

\begin{abstract}
Zero was a brilliant innovation and most significant achievement but it has threatened the foundations of modern physics and is behind all of the big puzzles in physics. Today, zero lies at the heart of one of the biggest scientific controversies of all time. The mysterious zero is posing challenges not only to intellectuals, philosophers, scientists, mathematicians, historians and theologizes but also is confusing and un-easing a common man. This article is devoted to raise awareness of social, conceptual and operational issues related with mysterious zero. The focus is those individuals involved in the process of teaching and learning mathematics. It is suspected that this article will enable them to think more clearly rather than to be in a state of confusion.
\end{abstract}

Key words: Avoidance, concept of infinity, division by zero, mysterious zero, Y2K.

\section{Introduction}

Invention of zero was a brilliant innovation and most significant achievement in present day mathematics. It was the last of the decimal digit to be established in numeral system. In contrast to the Roman numeral system, wherein zero was missing and arithmetic operations such as multiplication was difficult. It was the invention and introduction of zero in numeral system that has enabled to deal calculations with large number. It presents philosophical distinction between something and nothing. It helps clear up the philosophical questions about the difference: the presence of nothing, the absence of something, and the absence of everything. Zero is required for the law of non-contradiction. It is the absence of any given subject as regarding the respective predicate. It is the sum total of all of the quantitative forces in the universe [1]. Without the notion of zero, descriptive and prescriptive modeling processes in commerce, business and trade, astronomy, chemistry, mathematical sciences, etc. would have been almost impossible. Zero is a very unique number in nature. It is the only digit, which cannot stand alone. It needs some sort of companionship to give meaning to its life. By itself it is nothing. It is mathematically defined numerical function of nothing that is used not for an evasion but for an apprehension of reality. It is the only non-mystical nothing so far. It is a tool, a mathematical tool, for dealing with reality. Flourishment and advancement in present day science is largely facilitated because of invention and introduction of zero. It is a mathematical tool in sciences. It has increased the capability of computing skill even among the common people. Numeral system with zero is such an ingenious, efficacious, and conceptually integrated system that its enormous profundity, significance, and power is beyond human imagination. Being unique in meaning and function, it helped to express infinitely large numbers as well as making complex numerical operations much simpler. It was the power of zero that raised the curtains of science during the Renaissance, which 
had been drawn by the master neocheaters since 200BC [2]. Charles Seife [3], a science journalist, beautifully describes zero in his book as follow; it is a pivotal concept that has repeatedly revolutionized the foundations of civilization and philosophical thoughts. It is a strange number in the universe. The universe begins and ends with zero and is continuing its role as greatest paradoxes of human thoughts. It is not like other numbers, it is nothing and everything. Zero is the most fertile ideas that humanity has devised. It has shifted smoothly from history and philosophy to science and technology and is a gift for making complex ideas clear. It is an important tool of mathematics and has bedeviled and fascinated thinkers.

Despite all this, it has threatened the foundations of modern physics. Legendary thinkers like Pythagoras, Newton, Heisenberg and many today's astrophysicist, who has tried to understand zero and whose clashes shook the foundations of philosophy, mathematics, physics, history, science, and religion. It has pitted East against West and faith against reasons. Its intransigence persists in the dark core of black hole and the brilliant flash of the Big Bang [3].

Zero is behind all of the big puzzles in physics. In thermodynamics a zero became an un-crossable barrier: the coldest temperature possible. In Einstein's theory of general relativity, a zero became a black hole, a monstrous star that swallows entire suns and can lead us into new worlds. The infinite density of the black hole represents a division by zero. The big bang creation from the void is a division by zero. In quantum mechanics, the infinite energy of the vacuum is a division by zero and is responsible for a bizarre source of energy -- a phantom force exerted by nothing at all. Yet dividing by zero destroys the fabric of mathematics and the framework of logic and threatens to undermine the very basis of science. Today, zero lies at the heart of one of the biggest scientific controversies of all time. The mysterious zero is posing challenges not only to intellectuals, philosophers, scientists, mathematicians, historians and theologizes but also is confusing and un-easing a common man. There are more questions than being answered about mysterious zero like: Is it really something? Is it an abstract concept? Is it a number? What is zero? Who discovered it? How it is related with infinity (another mystery). Dilemma of the division by zero and many other operational confusions is there. Dilemma of Y2K. Is it negative or positive? Is it even or odd? Why electron is a zero-dimensional object. How one can explain zero factorial prior to the concept of permutation and Gamma function? Why zero is a noun, verb, adverb and adjective. Why Absolute zero (lowest possible temperature), translates to -273.15 degrees Celsius (or -459.67 degrees Fahrenheit). Why we try very often to avoid its pronunciation by many different substitute while it is not the case with other digits.

This article is devoted to raise awareness of issues related with mysterious zero. Historic perspective will not be highlighted. The focus is those individuals involved in the process of teaching and learning mathematics. It is suspected that this article will enable them to think more clearly rather than to be in a state of confusion. Before we discuss some other issues related with zero, I would like to emphasize here that reaching a solution by a short cut or may be too much depending on technology may lead us to a wrong approach/concept. Let us look at this example;

$$
x^{2}=1 \rightarrow x= \pm 1 \text { but } x^{2} \leq 1 \rightarrow x ?
$$

It will not be surprising if some students say $x \leq \pm 1$ because we are teaching them a short cut approach in the first solution. We should adopt as follow;

$$
x^{2}=1 \rightarrow x^{2}-1=0 \rightarrow(x-1)(x+1)=0 \rightarrow x= \pm 1
$$




$$
x^{2}=1 \rightarrow \sqrt{x^{2}}=\sqrt{1} \rightarrow|x|=1 \rightarrow \pm x=1 \rightarrow x= \pm 1 .
$$

\section{Social, Conceptual and Operational Issues}

\subsection{Avoidance}

In contrast to other digits or numbers, we very often intentionally or unintentionally practice a variety of avoidance for zero. Whether it is our telephone number, bank account, identification card number, student ID card, staff number, social security number, office number, level of a floor, street number and zip code, you just name anything, we try to avoid pronouncing zero and instead pronounce it as "oh", "naught", "duck", and "aught", etc. It is not the practice with other numbers, only special exception is for zero. Recently, 2000 was termed as $\mathrm{Y} 2 \mathrm{~K}$, avoidance. The reader might have noted another strange behavior of zero in English Literature, that is, it has been used as a verb, adverb, noun, and as adjective.

If we observe " 0 ", it appears at the end of computer's keyboard but at the bottom of calculator and telephone set. In some places it a starting point while at other places it is an ending point. One wonder, with these practices, is it possible to label zero as an end or a start. 0 on Celsius thermometer means different as compared to 0 on Fahrenheit thermometer. Use of zero as ground zero, as absolute zero temperature, as root ( $x$-intercept) of a function $f$ and as zero divisor structure in Real Algebra and Ring Theory etc. is another terminology of mysterious zero. We rarely denote any ground level as 0-level while in Spain, etc. they frequently use 0-level. Historically, it is also blamed that failure of Roman numeral system and so is the demise of Roman Empire was mostly due the absence of zero in their numeral system. Have we ever thought and observe of the confusions and fallacies in our own system? Zero has been avoided carefully in cardinality, ordinality, magnitude, length, and many other measurements. Julian calendar and Gregorian calendar were introduced in the absence of zero but now, with the present concept of zero, nothing much has been thought. Symbolically the vowel "o" and zero as a number " 0 " looks same, appears next to each other on computer keyboard but are treated quite differently. X-intercept, root, solution of a function is named as zero of a function. Our next issue is;

\subsection{Zero, a Concept and/or a Number}

Conceptuality is subjective while realization is objective. Conceptuality is mostly metaphysical while reality is physical. Concepts must be real in their correct context. The concept of zero was probably in use as early as human beings first began to do arithmetic [4], [5]. Concept of zero was derived from the concept of "void', "empty", "null", "nothing", "not there", "bottom", 'failure", "duck", etc. Philosophically, presence of "nothing" reflects "non-existence", absence of "something" reflects "non-availability" and absence of "anything" reflects "non-existence". Zero is a symbol for "not there" which is different from "nothing", "not there" reflects that number or item exist but that is not just available. Concept of zero was so important that it has given birth to negative numbers; otherwise we would have been dealing with only positive numerals as was the case in many old civilizations. Originally zero was not the candidate for being number. When the ideas of number became more and more abstract, philosophical thoughts shifted to science, it was then that makes possible the consideration of zero as a number. In different civilization, it appeared as part of their numeral system, while the other like Romans were not having the concept of zero. We conclude, "Zero is a concept as well as a number (digit)". It obeys most of the same rules of arithmetic that ordinary numbers do, so we call it a number. It is a unique and mysterious number because it does not obey all of the same rules as other numbers. We always mix up the abstract concept with concrete concept. Unfortunately, there is a lot of mix up and confusion because of these two concepts of zero. 


\subsection{Concept of Infinity}

To have a clear picture of many issues related to mysterious zero, we need to understand clearly about the concept of infinity, which within itself is another mystery. A proper understanding about infinity rests on a careful definition of what is finite? Otherwise we will be facing un-easing and confusing results. Unfortunately, starting from Brahmagupta till today, some of us try wrongly to express infinity in terms of zero [6]. Infinity is either endless cycle or an eternal limit. It is impossible to count or write out or express infinity through linear examples. Aristotle described infinity as; "It is plain, too, that the infinite cannot be an actual thing and a substance and principle. Mostly, the fallacy and confusion is due to our misunderstanding to a very crucial and important mathematical concept "equal to zero" and "approaching zero". Anyhow, it is agreed upon that "infinity is not a number" but rather "name of an abstract concept". Most often we also get confused that infinity is undefined while the fact is that infinity is a well-defined abstract concept but yes value of infinity is undefined. Interestingly, people are used to say that zero and infinity are two jokers in our numeral system.

\subsection{Multiplication by Zero}

Unfortunately, there are teachers who continue misleading students as follow:

$$
4(3)=\underbrace{3+3+3+3}_{4 \text { time }}=12 \quad n \times m=\underbrace{m+m+---+m}_{n \text { time }} \text { or } n \times m=\underbrace{n+n+---+n}_{m \text { time }}
$$

Therefore

$$
\infty \times 0=\underbrace{0+0+---+---}_{\infty \text { time }}=0 \text { or } \infty .
$$

More sophisticated arguments can also be made like: $\infty \times 0=\lim _{x \rightarrow \infty}\left(x \times \frac{1}{x}\right)=1$

Multiplication practice here is not justified. 0 is a number (digit) and $\infty$ is a concept. Multiplication is not meant to multiply a number (digit) with a concept. This type of writing should not be practice. This type of expression appears in indeterminate form like $\lim _{x \rightarrow 0^{+}}(x \ln x)$. Rather than writing as $0 \times \infty$, we should practice like $\lim _{x \rightarrow a}[f(x) g(x)]$ is in indeterminate form if $\lim _{x \rightarrow a} f(x) \rightarrow 0$ and $\lim _{x \rightarrow a} g(x) \rightarrow \infty$.

\subsection{Division by Zero}

Brahmagupta [7], an Indian mathematician, whom historians believed is a pioneer to introduce zero in Indian numeral system. He writes; "A number remains unchanged when divided by zero". He also writes "zero divided by zero is zero". Bhaskara [8], another Indian mathematician, even 500 years later writes; "A quantity divided by zero becomes a fraction the denominator of which is zero. This fraction in termed an infinite quantity". He has written $\frac{n}{0}=\infty$. One may observe the same concept in some of the current books of mathematics [Taylor III]. If we divide any number by zero on a calculator, the outcome would be an error "E". Division by zero produces a fatal condition in a computer system. Now, let us look at some of the common practices.

- $\frac{p}{q}=r \Leftrightarrow q r=p$, that is, inverting process of multiplication. 
- $\quad$ Dividing a number by another number means splitting it up into groups. For example: $\frac{8}{2}$ or $8 \div 2$ means that take 8 and divide it up between 2 groups.

- $\quad p$ is divisible by $q$ if $p$ can uniquely be written as $\mathrm{p}=\mathrm{bq}+\mathrm{r}$ where $0 \leq r<q$.

- Division is a fast subtraction, that is, $\frac{8}{2}$ or $8 \div 2$ is equivalent to $(8-2-2-2-2=0)$, that is, how many times 2 can be subtracted from 8 .

Does any of the approach or practice justify division by zero? It is a fallacy that any number divided by zero is infinity or subtracting zero infinite time from number results infinity. Zero is a number and infinity is an abstract concept. There is a difference between some variable equal to zero and a variable approaches zero. Division by zero is "meaningless", "undefined" in reals and there is no justification even to write $\frac{8}{0}$ or $8 \div 0$. Look at another concept $\frac{1}{0}=\infty \quad \& \frac{1}{\infty}=0 \Rightarrow 0 \bullet \infty=1$. Can infinity be the Inverse of zero?

Sometimes, we get confused in indeterminate forms, where the pivotal concept is the limit of a variable approaching zero or may be infinity. Involving division by a real quantity $\mathrm{x}$ which approaches zero may be in fact be well defined. A similar fallacy is to say that logarithmic value of zero is infinity $(-\infty)$ while the fact is that this operation is undefined. Similarly, zero is additive inverse of itself which is a unique property but other fact is that zero has no multiplicative inverse under any circumstances. Most of the indeterminate forms involve zero and/or infinity which we wrongly write and explain as; $\frac{0}{0}, \frac{\infty}{\infty}, \infty-\infty, 0^{0}, 0 \times \infty, \infty^{\infty}, \infty^{0}, 0^{\infty}$. Misconcepts are due to misunderstanding of zero, infinity, wrongly applying the binary operations and not differentiating between $x=0$ and $x \rightarrow 0$. We will discuss few cases;

- $\lim _{x \rightarrow 0^{+}}\left(\frac{1}{x}\right)=\infty$ because $\frac{1}{0}=\infty . \quad \frac{1}{0}=\infty$ does not qualify to write and declare it equal to infinity. We should say $\frac{1}{x} \rightarrow \infty$ as $x \rightarrow 0^{+}$but does not qualify to say equal to infinity. We should differentiate between limit and value of a function such as $f(x)=\frac{1}{x}$ then $f(x=0)=$ undefined .

- $\lim _{x \rightarrow-\infty} \frac{1}{x}=-0$ because $\frac{1}{-\infty}=-0$. Zero is neither positive nor negative so writing is wrong. Division is not justified because 1 is a number and infinity is an abstract concept. We should say; $\frac{1}{x} \rightarrow 0$ as $x \rightarrow-\infty$.

- $\infty+\infty=\infty$ : This is not an indeterminate form. Addition is not meant to add concept. We should say that if something approaches infinity and another thing also approached infinity and their sum is justified then their sum approached infinity.

- $\quad \frac{0}{0}$ : This is another dilemma of zero. Our understanding is that division by zero is an operational mistake, we should not divide by zero, and it is undefined (impossible). In this case, it is indeterminate form (not clear, uncertain, and inconclusive). To overcome this confusion we should say; The well defined fraction $\frac{x}{y}$ will be in indeterminate form if $x \rightarrow 0$ and $y$ also $\rightarrow 0$. 
Zero is behind all of the big puzzles in physics. In thermodynamics, zero is un-crossable barrier: "the possible coldest temperature". In Einstein's theory of general relativity, zero is black hole and infinite density of the black hole represents a division by zero. The big bang creation from the void is also division by zero. In quantum mechanics, the infinite energy of the vacuum is a division by zero (phantom force). General relativity and quantum mechanics, the two pillars of modern science have in common -- and what they clash over -- is zero." "The infinite zero of a black hole -- mass crammed into zero space, curving space infinitely -- punches a hole in the smooth rubber sheet. The equations of general relativity cannot deal with the sharpness of zero. In a black hole, space and time are meaningless.

\subsection{Zero Is Positive or Negative Number}

It took longer for the idea of negative numbers to be accepted. The negative sign along with the numbers is an extension of number system used to indicate directionality. Considering zero as starting point for both positive and negative numbers incorrectly confused that zero is positive as well as negative. The fact is +8 and -8 gives a sense of direction, that is, position to the right or left of zero while considering zero as a center or zero as a place between positive and negative. Another place where fallacy occurs is the interpretation of "non-negative" and "non-positive". The first include zero along with all positive numbers while the other include zero along with negative numbers. This is wrongly interpreted, as zero is both positive as well as negative without considering the differentiation between positive and non-negative and similarly negative and non-positive. Computers have two versions of zero; positive and negative. Another fallacy indicated in mathematics book is writing zero as +0 or -0 . The same appears in the context of additive inverse of zero wherein we think of negative zero as opposite of zero. The concept of opposite is our own managerial invention. For non-zero numbers, opposite may be understood as an equally distance number but on opposite side but in case of zero we are taking of opposite of a concept in term of number, that is. Quantity verses quality. To conclude our discussion, it is agreed upon that zero is neither positive nor negative [9].

\subsection{Misuse}

Since it is easy to change 0 to 6 or 9 so consequently we are forced to write figure in words.

\section{Conclusion}

There are many mix up due to distinct notion of mysterious zero, that is, zero as a number of our numeral system and zero as a concept for void, nothing, and not there, etc. There must be a clear understanding about abstract concept, concrete concept, and their application accordingly. The students trust heavily on their teachers and the textbooks. They trust to the extent that they are not willing to accept an argument from parents or other subject experts. This clearly indicates the important role of a teacher and recommended text in curricula. I feel that there is a need to defuse this trend and to project that objective of education is critical thinking rather than blind trust.

Many reference are there committing zero related mistakes, such as in the concept of; division by zero, evenness and/or oddness of zero, zero as positive or negative, equal to zero and approaching zero, indeterminate forms etc. Social avoidance of zero is another dilemma. Zero is a key player in our numeral system, misplacing it or misusing it or misunderstanding it will be a disastrous. These errors are not only linked to mathematics professionals, mathematics curricula, teachers or those related to mathematical disciplines but persist among their students, students of students etc. It is the case with common individuals who practice arithmetic. These errors are not limited to in class teaching and discussion but are also caused due to cultural impacts. The fast moving computer technology is playing its role. At the time of appointing a mathematics teacher, who have many other criteria but do not focus on the basic concepts of 
the subject? There are hardly any programs in practice to trained in-service teachers with the new approaches and concepts. I suspect, these mistakes and errors will continue from professionals to professionals, teachers to teachers, teachers to students and parents to children. With the help of current technology, messages regarding these errors can be conveyed in all circles. Not only this, mathematics, which is wrongly assumed as a boring subject, can be made more fun, more relevant, more exciting, more attractive and of course more challenging.

\section{References}

[1] Janet, L. (1998-1999). F6 Pure Mathematics Projects, St Francis' Canossian College, HK. Retrieved Dec. 2014 from http://www.sfcc.edu.hk/academic_subjects/mathematics/web/1998_1999_projects/Janet\%20Lo/Bb/ number.htm

[2] Russell, J. S. (2014).The Bible of the 3rd Millennium. Xlibris Corporation.

[3] Seife, C. (2000). Zero: The Biography of a Dangerous Idea. New York: Penguin Books.

[4] Barrow, J. D. (2001). The Book of Nothing. Vintage.

[5] Kaplan, R. (2000).The Nothing That Is: A Natural History of Zero. Oxford: Oxford University Press.

[6] David, F.W. (2004). Everything and More: A Compact History of Infinity. Norton, W.W. \& Company.

[7] Brahmagupta. (2014). Encyclopedia of World Biography. Retrieved from http://www.encyclopedia.com/doc/1G2-2550300030.html

[8] Salwi, D. M. (1986). Our Scientists. New Delhi: Children's Book Trust.

[9] James, V. D., James, R., \& Holli, A. (2009). Fundamentals of mathematics. Cengage Learning.

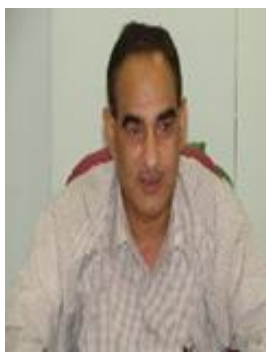

Mohammad Azram received his B.S. and M.Sc. (with distinction) degrees in mathematics from the University of Peshawar, Pakistan in 1974 and 1976 respectively. He started his career as a lecturer in the Department of Mathematics, University of Peshawar in Jan. 1977. He received his M.S. and Ph.D degree in mathematics from the University of Idaho (USA) in 1985 and 1989 respectively with CGPA 3.9/4.0. His research interest is in the area of low-dimensional cell-complexes/knot theory, point-set topology, mathematical physics and topological algebras. As a teacher, he served the University of Peshawar in the capacity of a lecturer, an assistant prof, Assoc. Prof. \& Prof. As a leader/administrator, he served the University of Peshawar as a director (Ph.D./M.Phil programs), the head (the Department of Computer Science) and a member (Academic Council, The Senate, and affiliation committee). He served the teaching community of Peshawar University as an elected member of Peshawar University Teacher's Association. He is associated with many journals such as Science International Lahore (the Managing Editor), Journal of Managerial Sciences, etc. 